\section{ATP synthase/apolipoprotéine A-I : un nouveau couple contre l'athérosclérose?}

Laurent Martinez, Sébastien Jacquet, François Tercé, Bertrand Perret, Xavier Collet, Ronald Barbaras
Inserm U.326,

CHU Purpan,

Place du Docteur Baylac,

31059 Toulouse Cedex 03,

France.

établir. Par ailleurs, on peut concevoir qu'à côté de ces protéines contrôlant les mouvements du cholestérol, intervienne(nt) un (des) récepteur(s) spécifique(s) de l'apoA-I (coopérant avec ces différentes protéines).

C'est dans ce contexte que nous venons de purifier et de caractériser un récepteur de haute affinité pour l'apoA-I, situé à la surface des hépatocytes [10]. Ce récepteur a été identifié comme étant la chaîne $\beta$ de l'ATP synthase, un complexe protéique majeur du feuillet interne de la mitochondrie. Sa localisation à la surface des hépatocytes humains a été confirmée par différentes approches expérimentales (microscopie confocale, cytofluorométrie...). Sa présence est associée à une activité ATP hydrolase à la surface cellulaire, suggérant que l'enzyme entière pourrait y être présente. Par ailleurs, nous avons observé que I'apoA-I stimule fortement l'endocytose des HDL. Or, cette endocytose apparaît strictement dépendante de l'ADP produit après stimulation de l'ATP synthase de surface par l'apoA-I. Ainsi, un nouveau rôle a été démontré pour une ATP synthase/hydrolase ectopique, capable de moduler les niveaux extracellulaires d'ADP et contrôlée par une apolipoprotéine plasmatique majeure, I'apoA-I. Pour évaluer l'implication physiologique de ces observations, des expériences ont été réalisées sur des foies de rat perfusés : un inhibiteur spécifique de l'activité hydrolase de I'ATP synthase, I'IF $F_{1}$, induit une diminution importante de l'internalisation des HDL par le foie, ce qui 
semble indiquer que, au moins chez les rongeurs, I'ATP synthase de surface est impliquée dans l'endocytose des HDL.

Ces travaux ont permis de démontrer que l'endocytose hépatique des HDL est contrôlée, dès le niveau membranaire, par l'activité ATP hydrolase de I'ATP synthase de surface, en amont de récepteurs purinergiques qui vont prendre ensuite le relais dans cette cascade de régulation de l'endocytose des HDL (Figure 1). L'ensemble de cette nouvelle voie métabolique ajoute un élément important, sorte de «chaînon manquant», à la compréhension des mécanismes contrôlant la dynamique du transport-retour du cholestérol et susceptibles de devenir, dans un proche avenir, des cibles pharmacologiques de choix pour la modulation de I'homéostasie du cholestérol.

La présence de l'ATP synthase au niveau de la membrane plasmique a déjà été rapportée dans des cellules endothéliales humaines $[11,12]$, où elle serait un récepteur de l'angiostatine, suggérant son implication dans l'angiogenèse. L'ATP synthase a également été localisée à la surface de certaines lignées tumorales lymphocytaires [13], pouvant ainsi constituer un marqueur de tumorigénicité.

Mais la présence inattendue, à la surface de plusieurs types cellulaires, de I'ATP synthase pose maintenant la question des mécanismes permettant cette localisation membranaire, et laisse entrevoir que les mitochondries pourraient jouer un rôle nouveau, insoupçonné à ce jour. Par ailleurs, elle relance la question des rôles physiologiques de l'apoA-I, protéine abondante dans le plasma et qui, comme ligand de cette ATP synthase membranaire, pourrait intervenir dans divers processus physiopathologiques tels que l'athérosclérose ou l'angiogenèse. $\vartheta$

ATP synthase and apolipoprotein synthase: new players in atherosclerosis

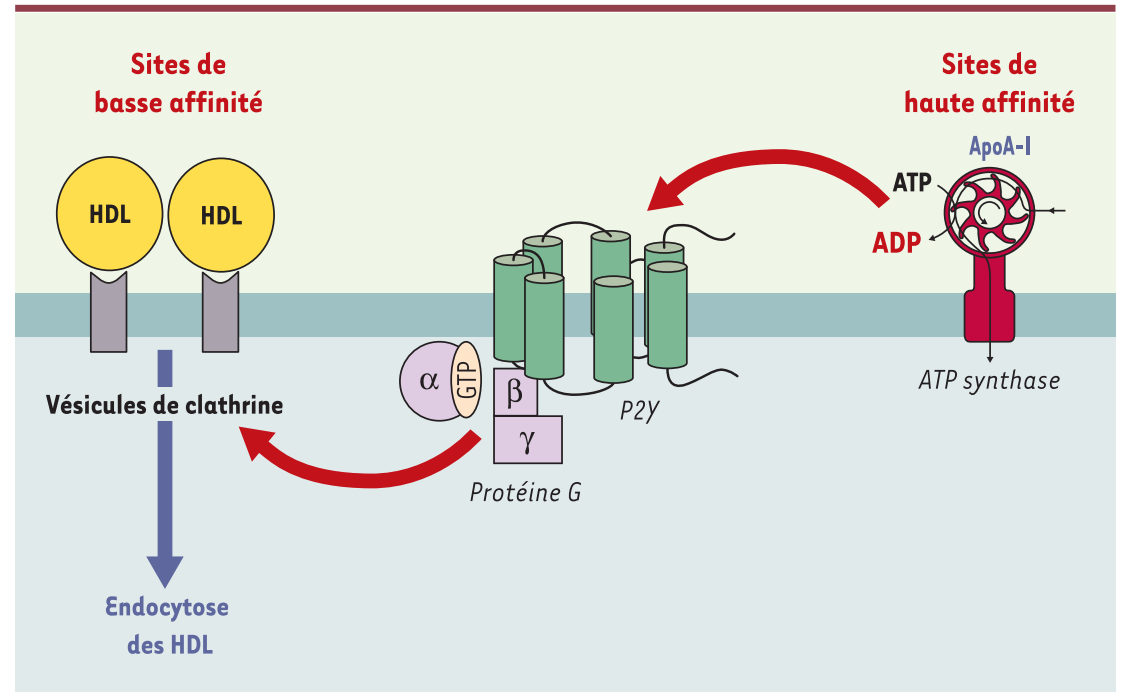

Figure 1. Intervention de l'ATP synthase dans l'endocytose des HDL.

\section{RÉFÉRENCES}

1. Assmann G, Carmena R, Cullen $\mathrm{P}$, et al. Coronary heart disease: reducing the risk: a worldwide view. International task force for the prevention of coronary heart disease. Circulation 1999 ; 100 : 1930-8.

2. Rhoads GG,

Gulbrandsen CL, Kagan A. Serum lipoproteins and coronary heart disease in a population study of Hawaii Japanese men. $N$ Engl J Med 1976 ; 294 : 293-8.

3. Matsumoto A, Mitchell A, Kurata $\mathrm{H}$, et al. Cloning and characterization of HB2, a candidate high density lipoprotein receptor. Sequence homology with members of the immunoglobulin superfamily of membrane proteins. J Biol Chem 1997 ; 272 : 16778-82.

4. Bocharov AV, Vishnyakova TG, Baranova IN, et al. Characterization of a $95 \mathrm{kDa}$ high affinity human high density lipoproteinbinding protein. Biochemistry 2001 ; 40 : 4407-16.
5. Acton S, Rigotti A, Landschulz KT, et al. Identification of scavenger receptor SR-BI as a high density lipoprotein receptor. Science 1996 ; 271 : 518-20.

6. Trigatti B, Rayburn H, Vinals $M$, et al. Influence of the high density lipoprotein receptor $\mathrm{SR}-\mathrm{BI}$ on reproductive and cardiovascular pathophysiology. Proc Natl Acad Sci USA 1999 ; 96 : 9322-7.

7. Chambenoit 0 , Hamon $y$, Marguet D, et al. Specific docking of apolipoprotein $A-I$ at the cell surface requires a functional $A B C A 1$ transporter. J Biol Chem 2001 ; 276 : 9955-60.

8. Rigot V, Hamon $y$, Chambenoit 0 , et al. Distinct sites on ABCA control distinct steps required for cellular release of phospholipids. J Lipid Res 2002 ; 43 : 2077-86.

9. Groen AK, Bloks VW, Bandsma RH, et al. Hepatobiliary cholesterol transport is not impaired in Abcal-null mice lacking HDL. J Clin Invest 2001 ; $108: 843-50$.
10. Martinez LO, Jacquet $S$, Esteve JP, et al. Ectopic beta-chain of ATP synthase is an apolipoprotein A-I receptor in hepatic $\mathrm{HDL}$ endocytosis. Nature 2003 ; 421 : 75-9.

11. Moser TL, Stack MS, Asplin I, et al. Angiostatin binds ATP synthase on the surface of human endothelial cells. Proc Natl Acad Sci USA 1999 ; 96: 2811-6.

12. Moser TL, Kenan DJ, Ashley TA, et al. Endothelial cell surface FlFO ATP synthase is active in ATP synthesis and is inhibited by angiostatin. Proc Natl Acad Sci USA 2001 ; 98 : 6656-61.

13. Das B, Mondragon M0, Sadeghian $M$, et al. A novel ligand in lymphocytemediated cytotoxicity: expression of the beta subunit of $\mathrm{H}^{+}$transporting ATP synthase on the surface of tumor cell lines. J Exp Med 1994 ; $80: 273-81$. 